\title{
Lesión osteoblástica en columna cervical de un paciente pediátrico
}

Osteoblastic lesion in the cervical spine of a pediatric patient

\author{
Dr. José Manuel Reyes-Hernández, ${ }^{*}$ \\ Dr. Fernando Ramos-Morales, ${ }^{\ddagger}$ Dr. David Cruz-Guillen ${ }^{\S}$ \\ Hospital Regional «Lic. Adolfo López Mateos» ISSSTE. México. \\ * Médico Adscrito al servicio de Ortopedia, Titular del Módulo de Ortopedia Pediátrica. \\ ₹ Médico Adscrito al servicio de Ortopedia, Titular del Módulo de Cirugía de Columna. \\ $\S$ Médico Adscrito al servicio de Ortopedia, Titular del Módulo de Tumores Óseos.
}

\section{RESUMEN}

El osteoblastoma es un tumor óseo benigno raro. Aunque las características histológicas en la mayoría de los casos son distintivas, hay varias mutaciones que hacen que el diagnóstico sea desafiante. Puede simular una variedad de otros tumores óseos benignos, pero lo más importante es distinguir el osteoblastoma del osteosarcoma, lo que puede ser difícil.

Palabras clave: Osteoblastoma, niños, osteosarcoma, tumores.

Nivel de evidencia: IV

\section{ABSTRACT}

Osteoblastoma is a rare benign bone tumor. Although the histologic features in most cases are distinctive, there are various permutations that make the diagnosis challenging. It can mimic a variety of other benign bone tumors, but more importantly, distinguishing it from osteoblastoma like osteosarcoma can be difficult.

Keywords: Osteoblastoma, children, osteosarcoma, tumors.

Evidence level: IV

El osteoblastoma es un tumor solitario, benigno, formador de hueso y tejido osteoide, bien diferenciado y normalmente tiene un estroma vascular. ${ }^{1}$ Presenta una incidencia inferior a $1 \%$ de todos los tumores del hueso y de $3-3.5 \%$ de los tumores óseos benignos. ${ }^{5-7}$ Es más frecuente en hombres que en mujeres, con una proporción de 2:1 y con incidencia máxima entre 15 y 20 años de edad. ${ }^{6}$ Afecta preferentemente a la columna vertebral y los huesos largos; y con menos frecuencia la pelvis,
Inicialmente fue descrito por Jaffe y Mayer ${ }^{1}$ en 1932 bajo el nombre descriptivo de «un tumor formador de tejido osteoide osteoblástico en un hueso metacarpiano». Más tarde, Jaffe $^{2}$ y Lichtenstein ${ }^{3}$ en 1956, y Lichtenstein y Sawyer ${ }^{4}$ en 1964 describieron osteoblastomas en varios artículos bajo el título de «osteoblastoma benigno». 
la escápula, las costillas y la clavícula. ${ }^{8}$ Las manifestaciones varían según la situación y tamaño del tumor. ${ }^{5}$

Para establecer el diagnóstico, las radiografías simples pueden variar de acuerdo con el estadio del tumor en el momento de la presentación. 2,9 Para cada tipo de tumor los autores consideran características clínicas, apariencias radiológicas, patología macro y microscópica, tratamiento y pronóstico para su completo estudio. ${ }^{10}$ Por lo general las lesiones aparecen como imágenes radiolúcidas expansivas bien definidas, a veces asociadas con trabéculas internas delgadas con márgenes escleróticas ${ }^{11,12}$ Las lesiones son por lo regular mayores de $2 \mathrm{~cm}$ y llegan hasta $8 \mathrm{~cm}$ de diámetro. Esto es generalmente más grande que la presentación de un osteoma osteoide. . $^{2,12} 13$

El tratamiento del osteoblastoma debe ser quirúrgico mediante curetaje intralesional o resección en bloque. ${ }^{14}$

\section{PRESENTACIÓN DEL CASO}

Se trata de paciente femenino de ocho años, sin antecedentes de importancia previos a su padecimiento, con presencia de tumoración con datos clínicos de un comportamiento biológico activo.

$\mathrm{Al}$ interrogatorio dirigido, refiere dolor EVA 6/10 en región posterior de cuello asociado a actividad física diaria, acompañado de rigidez matutina de tres semanas de evolución, sin síntomas de compromiso neurológico ni radicular, sin limitación a los movimientos del cuello en todos sus ejes ni antecedentes traumáticos (Figura 1).

A la exploración física dirigida se aprecian arcos de movilidad de columna cervical conservados para todos los ejes, a la hiperflexión del cuello se observa aumento de volumen en zona paravertebral derecha, sin evidencia de

Figura 1:

Deformidad evidente en columna cervical vista lateral.
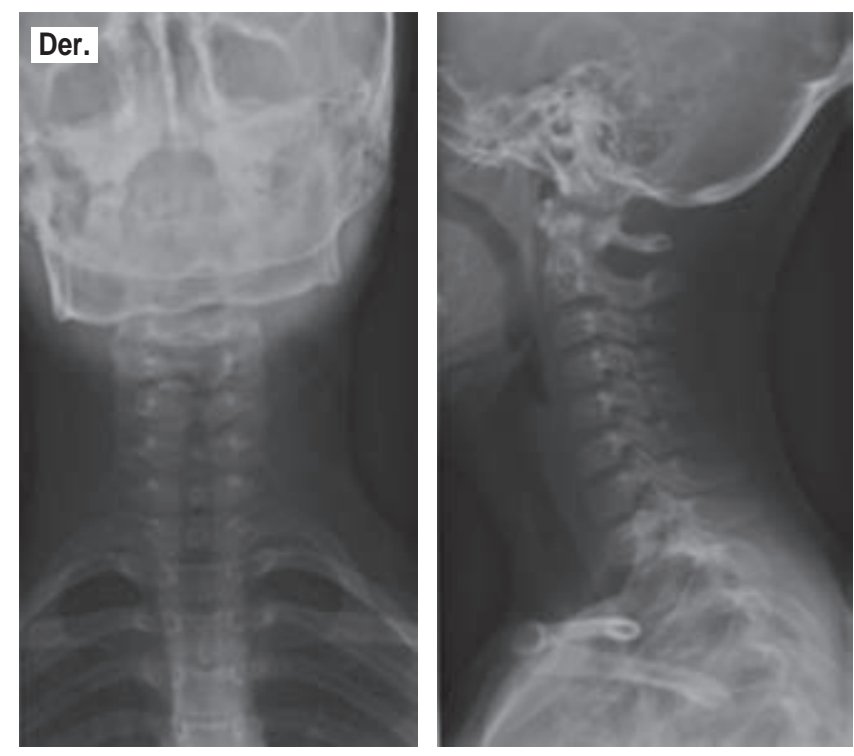

Figura 2: Radiografía anteroposterior y lateral de columna cervical observándose lesión única sobre lámina derecha de quinto cuerpo vertebral cervical.

red venosa colateral y sin cambios de coloración tegumentaria. Se palpa tumoración sólida de aproximadamente 2 $\mathrm{cm}$ de diámetro, adherida a planos profundos, con dolor a la palpación, sin irradiación del dolor ni cambios dérmicos locales, extremidades superiores con arcos de movilidad para todas las articulaciones íntegros, con fuerza y sensibilidad conservadas y llenado capilar distal inmediato.

Se obtienen estudios radiográficos en proyecciones anteroposterior y lateral de columna cervical (Figura 2), observando lordosis fisiológica, cuerpos vertebrales con conservación de su altura, adecuados espacios articulares, sin datos de listesis; se observa lesión única a nivel de lámina derecha de quinta vértebra cervical, radiopaca, de bordes bien definidos, de $20 \times 15 \mathrm{~mm}$ aproximadamente que no infiltra tejidos adyacentes, sin reacción perióstica ni extensión extraósea.

Se solicitan estudios de analítica sanguínea, en los que se encuentra fosfatasa alcalina de 312 unidades/litro que se considera normal por actividad ósea fisiológica; ultrasonografía con reporte de lesión sólida de componente óseo paracervical derecho, probable osteocondroma; tomografía axial computarizada simple donde se aprecia imagen hiperdensa en lámina derecha de quinto cuerpo vertebral, única, de aproximadamente $15 \times 20 \mathrm{~mm}$ (Figura 3); resonancia magnética nuclear simple que reporta imagen hiperintensa que rebasa bordes, de aproximadamente $15 \times 20 \mathrm{~mm}$, en lámina derecha de quinto cuerpo vertebral (Figura 4) y gammagrafía ósea con MDP que indica actividad osteoblástica con discreto aumento en quinto cuerpo vertebral cervical principalmente lado derecho, no se observan zonas de infiltración ósea. 
Se realiza biopsia escisional de tumoración mediante abordaje posterior en columna cervical (Figura 5), se observa una lesión sólida de aproximadamente $15 \times 20$ $\mathrm{mm}$, sin infiltración de tejidos adyacentes y sin datos de reacción perióstica, cortical y bordes bien definidos, base en lámina derecha de quinta vértebra cervical, que verifica no interferencia en movilidad de articulación proximal y distal, la lesión se reseca desde su base permitiendo margen de $5 \mathrm{~mm}$, sin invasión intradural y sin afectar estabilidad de lámina vertebral, la cual se observa íntegra, por lo que no requiere estabilización quirúrgica. Se envía muestra a estudios histopatológicos.

Clínicamente la paciente cursa con buena evolución postquirúrgica, neurológicamente íntegra.

Durante seguimiento en consulta externa la paciente refiere mejoría del dolor y disminución de la rigidez. Funcionalmente se establece escala funcional Karnofsky 100/100 postquirúrgico y prequirúrgico de 60-80/100 (Figura 6).
Resultados de estudios de histopatología de tumor en lámina derecha de quinta vértebra cervical con reporte de tejido óseo, médula ósea y fibrosis sin atipia celular con datos compatibles de osteoblastoma (Figura 7).

\section{DISCUSIÓN}

El osteoblastoma es raro y representa sólo 1\% de los tumores óseos primarios. Tiene un amplio rango de edad (6-75 años). Sin embargo, con mayor frecuencia afecta a adolescentes y adultos jóvenes (edad media 20 años). ${ }^{11}$

El osteoblastoma no tiene una presentación clínica específica, sus manifestaciones clínicas varían según la situación y el tamaño del tumor. El paciente se queja principalmente de dolor progresivo localizado en el área de la lesión. Este dolor a veces aumenta por la noche, y no se calma con aspirina, a diferencia de lo que ocurre con el osteoma osteoide. En otros casos puede
Figura 3:

Tomografía axial computarizada

de columna cervical donde se observa imagen en lámina derecha en quinta vértebra cervical única.
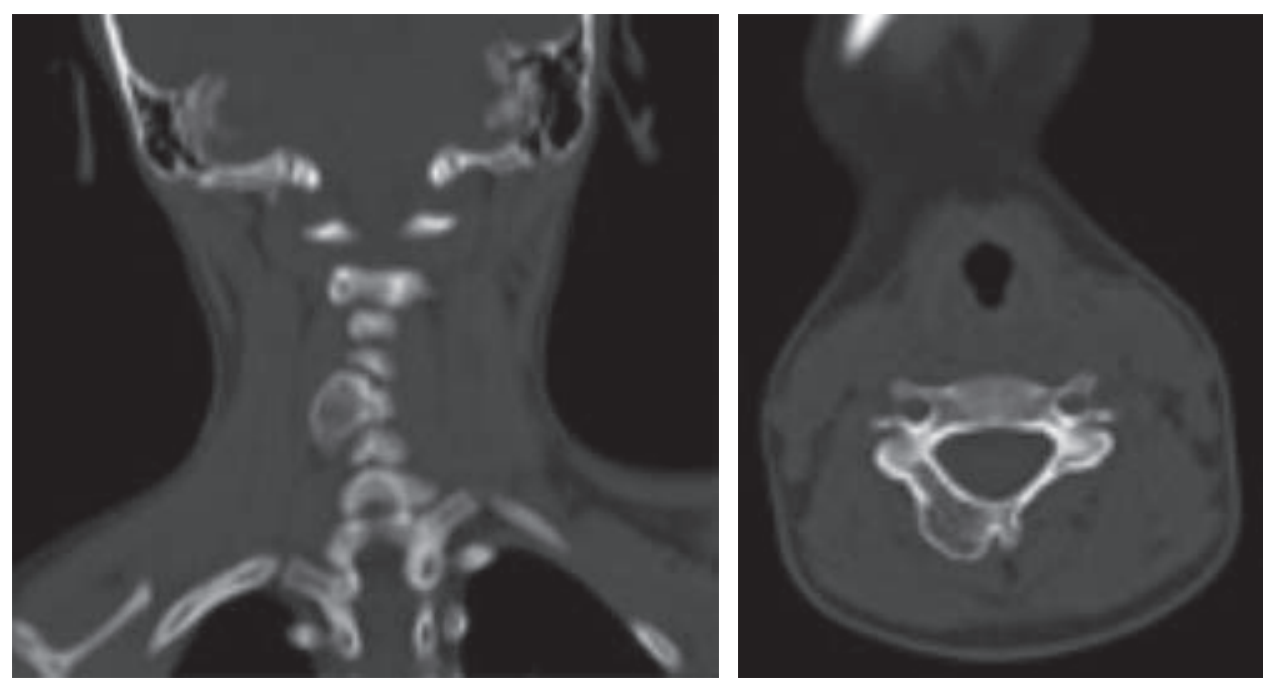
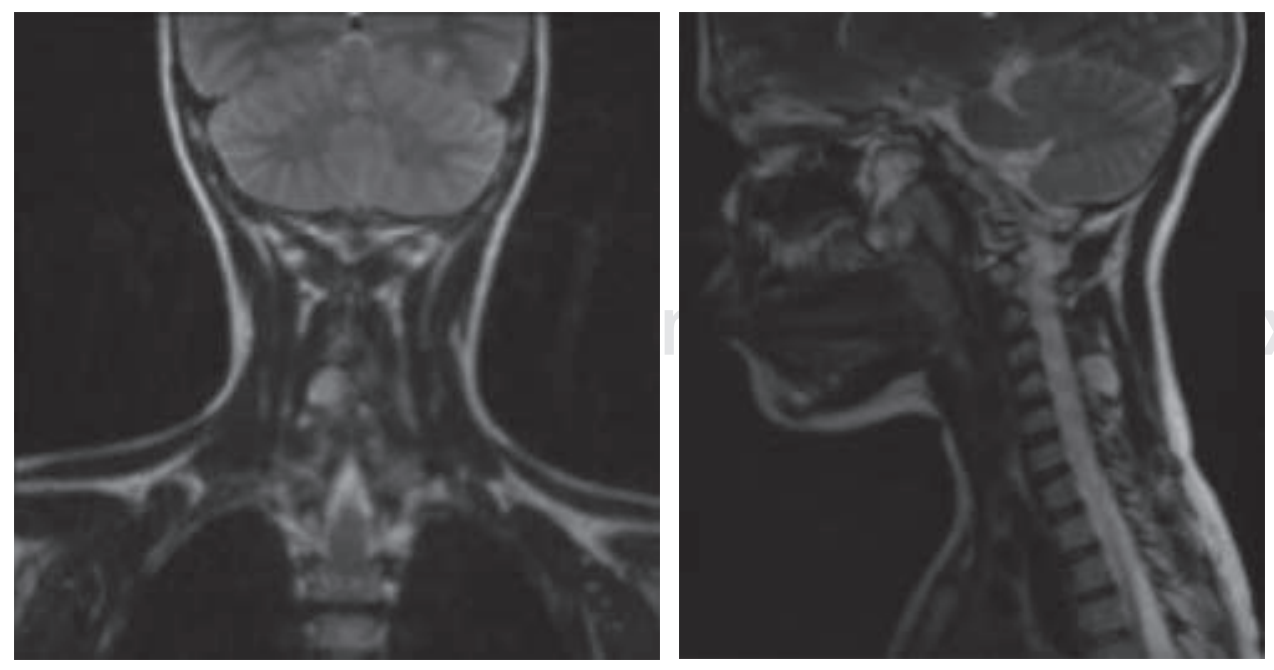

Figura 4:

Resonancia magnética simple. Imagen hiperintensa que rebasa bordes de aproximadamente $15 \times 20 \mathrm{~mm}$, lámina derecha quinto cuerpo vertebral. 

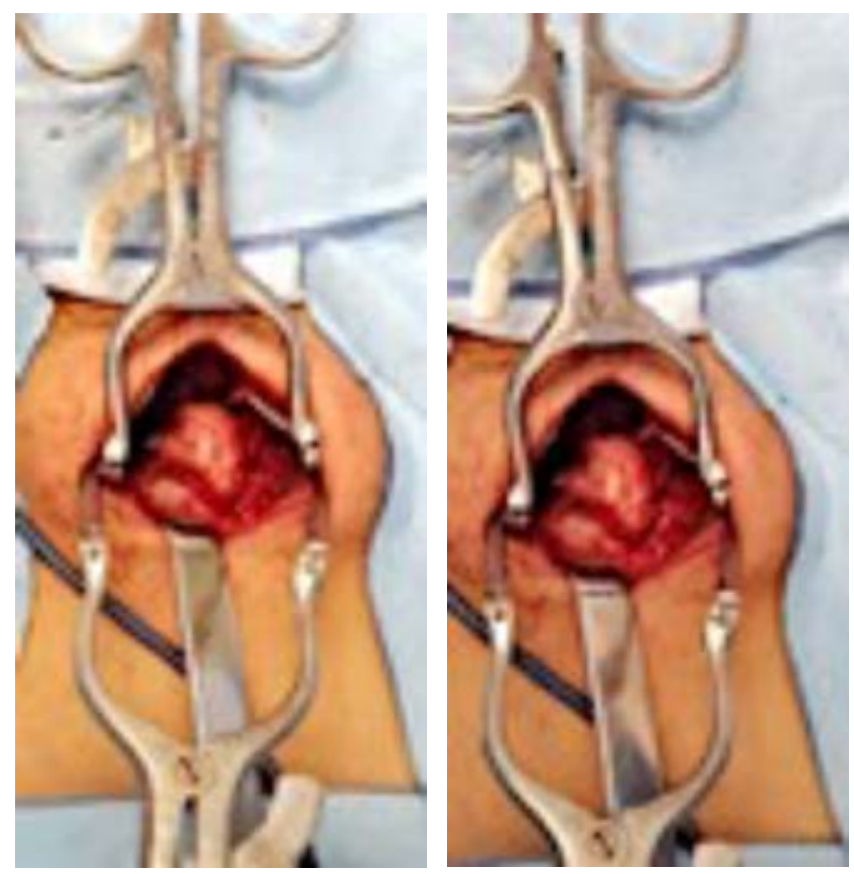

Figura 5: Toma de biopsia escisional de tumoración sobre lámina de la quinta vértebra cervical.

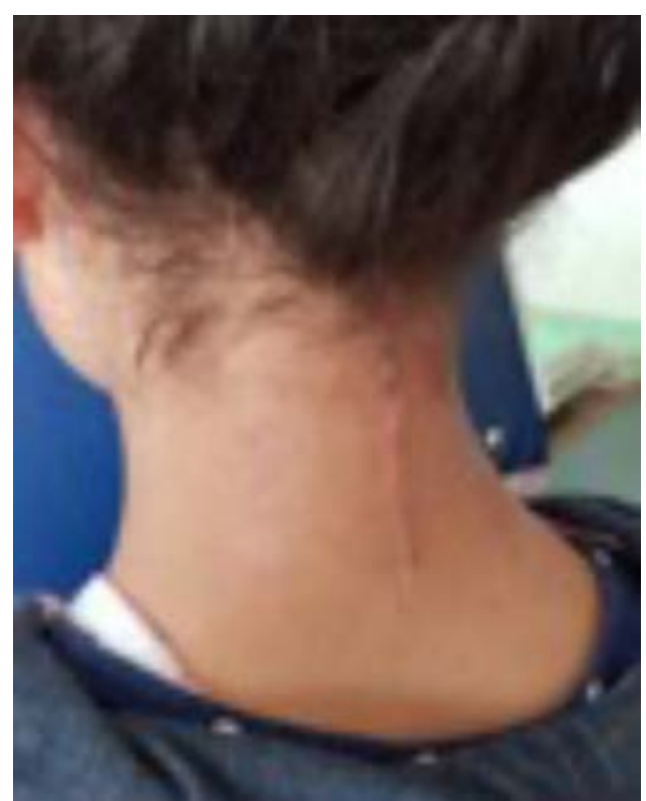

Figura 6: Fotografía postquirúrgica con dos semanas de evolución posterior a la cirugía.

deformar el hueso afectado y aparecer como una masa palpable, o puede ser asintomático y detectado en forma accidental. ${ }^{5}$

McLeod, Dahlin y Beabout clasificaron todas las lesiones menores de $1 \mathrm{~cm}$ de diámetro como osteomas

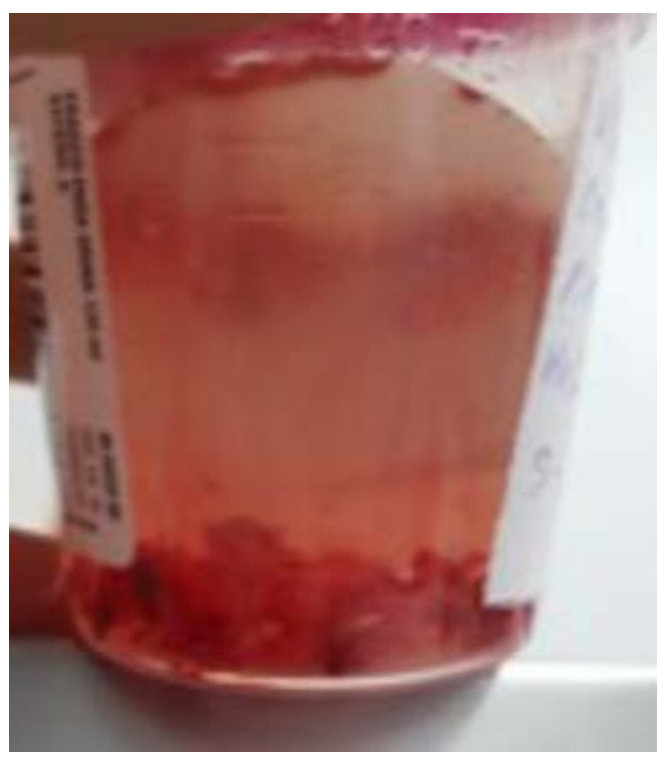

Figura 7: Aspecto macroscópico de la tumoración de quinto cuerpo vertebral.

osteoides y todos aquellos mayores de $2 \mathrm{~cm}$ como osteoblastomas. Entre 1 y $2 \mathrm{~cm}$, la diferenciación puede ser difícil.

Su estudio incluye 123 pacientes con osteoblastoma. Casi 90\% tenía menos de 30 años; casi tres cuartas partes estaban en la segunda y tercera décadas.

El síntoma cardinal, el dolor, ocurrió en $84 \%$ de los pacientes y por lo general se localizó en el sitio del tumor, excepto cuando el tumor incidió en las raíces nerviosas adyacentes o en la médula espinal. En 13\% de los pacientes el dolor fue peor por la noche. La aspirina alivió el dolor en sólo 7\%.

También un hallazgo común fue hinchazón localizada. Los pacientes con tumores espinales con frecuencia tenían escoliosis y espasmo muscular; los síntomas neurológicos fueron comunes en este grupo (26\%). La atrofia por desuso y la claudicación también ocurrieron en las extremidades. ${ }^{15}$

Marsh y colaboradores ${ }^{9}$ reportaron 25 casos de osteoblastoma, de los cuales sólo uno fue en la mano a nivel del segundo metacarpiano; son tumores por lo regular con un tamaño mayor de $2 \mathrm{~cm}$ en comparación de osteoma osteoide que particularmente son más pequeños, también la localización del osteoma osteoide es diferente.

Jackson informó $10 \%$ de recurrencia después de una resección incompleta. ${ }^{16}$ Marsh y asociados ${ }^{9}$ y Lucas y sus colegas $^{11}$ recolectaron 197 y 305 casos, respectivamente, e informaron recidiva local en 16 a $21 \%$ de los casos. El tamaño, la ubicación y la capacidad para resecar toda la lesión son factores importantes relacionados con la recurrencia, la morbilidad y la mortalidad, aunque la metástasis rara vez se ha documentado. ${ }^{11,13}$ 


\section{CONCLUSIONES}

De acuerdo con la literatura, la incidencia y presentación de esta patología es poco frecuente, supone $1 \%$ de todos los tumores óseos primarios. Predomina en pacientes jóvenes (10-25 años) y su prevalencia es mayor en varones (2:1), motivo por el que es importante reconocer a tiempo este tipo de padecimiento en los pacientes pediátricos y ser oportuno en su diagnóstico y tratamiento.

Debido a su rareza de este tipo de tumores, Moon KS y cols también reportaron una tumoración de un osteoblastoma benigno que surgió en el hueso occipital y que recomendaban su seguimiento por su alta posibilidad de recurrencia. ${ }^{17}$

El diagnostico diferencial de este tipo de tumores debe incluir tumor de celulas gigantes, osteosarcoma, quiste óseo aneurismatico, encondroma y condrosarcoma. ${ }^{18}$

\section{REFERENCIAS}

1. Jaffe HL, Mayer L. An osteoblastic osteoid tissue forming tumor of the metacarpal bone. Arch Surg. 1932; 24: 550-564.

2. Jaffe HL. Benign osteoblastoma. Bull Hosp Jt Dis. 1956; 1: 141-151.

3. Lichtenstein L. Benign osteoblastoma: a category of osteoid- and bone-forming tumors other than classical osteoid osteoma which may be mistaken for giant-cell tumor or osteogenic sarcoma. Cancer. 1956; 9(5): 1044-1052.

4. Lichtenstein L, Sawyer WR. Benign osteoblastoma: further observations and report of twenty additional cases. J bone Joint Surg Am. 1964; 46: 755-765.

5. Greenspan A, Remagen W. Differential diagnosis of tumors and tumor-like lesions of bone and joints. Philadelphia: LippincottRaven; 1998.
6. Masquijo J, Schumacher F, Kontio K. Osteoblastoma costal infantil. Informe de un caso y revisión de la bibliografía. Rev Asoc Argent Ortop Traumatol. 2011; 76(16): 8-171.

7. Villalobos CE, Rybak LD, Steiner GC, Wittig JC. Osteoblastoma of the sternum: case report and review of the literature. Bull NYU Hosp Jt Dis. 2010; 68(1): 55-59.

8. Rocca CD, Huvos AG. Osteoblastoma: varied histological presentations with a benign clinical course: an analysis of 55 cases. Am J Surg Pathol. 1996; 20(7): 841-850.

9. Marsh BW, Bonfiglio M, Brady LP, Enneking WF. Benign osteoblastoma: range of manifestations. J Bone Joint Surg Am. 1975; 57: 1-9.

10. Huvos AG. Osteoblastoma. In: Bone tumors: diagnosis, treatment and prognosis. 2nd ed. Philadelphia: WB Saunders; 1991. pp. 67-83.

11. Lucas DR, Unni KK, McLeod RA, O'Connor MI, Sim FH. Osteoblastoma: clinicopathologic study of 306 cases. Hum Pathol. 1994; 25(2): 117-134.

12. Frassica FS, Waltrip RL, Sponseller PD et al. Clinicopathologic features and treatment of osteoid osteoma and osteo- blastoma in children and adolescents. Orthop Clin North Am. 1996; 27(3): 559-574.

13. Golant A, Lou J, Erol B et al. Pediatric osteoblastoma of the sternum: a new surgical technique for reconstruction after removal. Case report and review of literature. J Pediatr Orthop. 2004; 24(3): 319-322.

14. Atesok K, Alman B, Schemitsch E, Peyser A, Mankin H. Osteoid osteoma and osteoblastoma. J Am Acad Orthop Surg. 2011; 19: 678-689.

15. McLeod RA, Dahlin DC, Beabout JW. The spectrum of osteoblastoma. AJR Am J Roentgenol. 1976; 126(2): 321-325.

16. Jackson RP. Recurrent osteoblastoma: a review. Clin Orthop Relat Res. 1978; (131): 229-233.

17. Moon KS, Jung S, Lee JH et al. Benign osteoblastoma of the occipital bone: case report and literature review. Neuropathology. 2006; 26: 141-146.

18. Sforzo CR, Scarborough MT, Wright TW. Bone-forming tumors of the upper extremity and Ewing's sarcoma. Hand Clin. 2004; 20: 303-315. 